\title{
Limitations in photosynthesis of sugar beets under water deficit and rehydration conditions
}

\author{
Yangyang $\mathrm{Li}^{1}$, Cong $\mathrm{Fei}^{1}$, Jixia $\mathrm{Su}^{1}$, Ningning Liu ${ }^{1}$, Fuyu $\mathrm{Ma}^{1}$, Hua Fan ${ }^{1}$, and Kaiyong \\ Wang $^{1}$ \\ ${ }^{1}$ Shihezi University
}

October 29, 2020

\begin{abstract}
The limiting factors in photosynthesis for many crops under water deficit and rehydration conditions have been studied, but few studies of this kind on sugar beet can be found.Our study aimed to analyze the dynamic changes of mesophyll limitations, stomatal limitations, and biochemical limitations by examining the influences of water deficit and rehydration on photosynthetic characteristics, electron transport, and leaf anatomical structure. According to the analysis on photosynthetic characteristics, severe deficit irrigation treatment significantly decreased photosynthesis rate, light saturation point, and CO2 saturation point. After rehydration, there was no difference in photosynthetic characteristics between the well irrigated sugar beets and the ones with severe deficit irrigation treatment. The analysis on leaf anatomical structure found that sugar beets dealt with water deficit by increasing leaf thickness, and the severe deficit irrigation treatment significantly decreased the number of chloroplasts without influencing each one in terms of shape and cross-section area. Therefore, deficit irrigation treatments cannot significantly influence photosynthetic assimilation of sugar beets. The analysis of limitations in photosynthesis showed that the stomatal and biochemical limitations increased while the mesophyll limitations decreased when sugar beets were under water deficit; however, the stomatal and biochemical limitations decreased while the mesophyll limitations increased after rehydration.
\end{abstract}

\section{Hosted file}

Manuscript .pdf available at https://authorea.com/users/371310/articles/489678-limitations-inphotosynthesis-of-sugar-beets-under-water-deficit-and-rehydration-conditions

\section{Hosted file}

Figiures.pdf available at https://authorea.com/users/371310/articles/489678-limitations-inphotosynthesis-of-sugar-beets-under-water-deficit-and-rehydration-conditions 